\title{
El laboratorio como un espacio para propiciar el aprendizaje significativo para los cursos por competencias de análisis cuantitativo en nivel medio superior y de química analítica ii del nivel superior utilizando el modelo didáctico la actividad integradora como herramienta
}

\author{
Judith Rocha Gamez \\ jrocha05@hotmail.com \\ Escuela y Preparatoria Técnica Medica UANL, \\ Monterrey Nuevo León México \\ Graciela Granados Guzmán \\ graciela.granadosgu@uanl.edu.mx
}

Facultad de Medicina UANL, Monterrey Nuevo León México

\section{RESUMEN}

Cuando se imparten asignaturas curriculares bajo la estrategia, además del objetivo que se pretende que el alumno aprenda a aprender (Estevez 2002) y tenga determinadas actitudes y habilidades para lograr aprendizajes significativos (Ausubel), también es necesario establecer diferentes estrategias para Ser capaz de evaluar pedagógicamente el desempeño del alumno.

El modelo de enseñanza aquí presentado permite diseñar la enseñanza como base del uso de estrategias cognitivas (Estevez 2002)

En cuanto a las condiciones involucradas en el modelo, estas son la participación activa del alumno: mediación, orientación y seguimiento del aprendizaje por parte del docente; aprovechar los errores y la retroalimentación como fuentes de aprendizaje y secuenciar la enseñanza para obtener el conocimiento de la meta (Estevez 2002)

El requisito de que los estudiantes logren sus logros individualmente y solo a través de sus propios esfuerzos es manejarnos a nosotros mismos bajo el paradigma de la teoría del aprendizaje por competencias (Barr 1995).

Las asignaturas como análisis cuantitativo de los planes de estudio del Técnico en Laboratorio Clínico y Química Analítica en la licenciatura de Biólogo Químico Clínico, tienen en común que son parte del proceso que utiliza diferentes métodos para analizar una muestra y cuantificar sus componentes, para que son cursos teóricos / prácticos.

Para evaluar plenamente al alumno, se diseña una actividad integradora para cada curso, que consta de lo siguiente: 
El alumno volverá a los conceptos aprendidos en sus cursos anteriores tales como:

Química I y II, Matemáticas, Análisis Cualitativo y Cuantitativo y en el caso de Análisis

Cualitativo para el Técnico en Laboratorio Clínico y las asignaturas de: Química General y Química Analítica I en el caso de Química Analítica II del QCB.

Palabras clave: estrategia, utilización, autoevaluación, habilidades, habilidades 


\section{ABSTRAC}

When curricular subjects are taught under the strategy, in addition to the aim that the student is intended to learn to learn (Estevez 2002) and have certain attitudes and skills to achieve meaningful learning (Ausubel), it is also necessary to establish different strategies to be able to evaluate pedagogically the student's performance.

The teaching model presented here allows to design teaching as the basis of the use of cognitive strategies (Estevez 2002)

As for the conditions involved in the model, these are the active participation of the student: mediation, guidance and monitoring of learning by the teacher; leveraging errors and feedback as sources of learning and sequencing teaching to obtain the goal knowledge (Estevez 2002)

The requirement that students achieve their achievements individually and only through their own efforts is to manage ourselves under the paradigm of competency learning theory (Barr 1995)

The subjects as quantitative analysis of the curricula of the Technician in Clinical Laboratory and Analytical Chemistry in the bachelor's degree of Clinical Chemical Biologist, have in common that they are part of the process that uses different methods to analyze a sample and quantify its components, for which they are theoretical /practical courses.

To fully evaluate the student, an integrative activity is designed for each course, which consists of the following:

The student will return to the concepts learned in his past courses such as: Chemistry I and II, Mathematics, Qualitative and Quantitative Analysis and in the case of Qualitative Analysis for the Technician in Clinical Laboratory and the subjects of: General Chemistry and Analytical Chemistry I in the case of Analytical Chemistry II of the QCB.

Keywords: Strategy, Utilization, Self-Assessment, Skills, Skills

Artículorecibido: 25 enero 2021

Aceptado para publicación: 28 febrero2021

Correspondencia: jrocha05@hotmail.com

Conflictos de Interés: Ninguna que declarar 


\section{INTRODUCCIÓN.}

La labor de todo maestro es lograr que sus alumnos aprendan, en una forma razonada e integral, es decir no sólo limitarse a que memoricen los contenidos, sino que relacionen y apliquen los conceptos adquiridos en la resolución de situaciones que se le presenten en el futuro, llevándose así un proceso mental más profundo que garantiza que no fácilmente se olviden los conceptos.

Lograr lo anterior es una tarea titánica, que no sólo involucra el trabajo del maestro en el aula, sino también un conjunto de especialistas, como son pedagogos, psicólogos, metodólogos, sociólogos etc. quienes trabajan planeando, diseñando y evaluando todo el contexto en el que está implicado tanto el maestro como el alumno.

Es importante señalar que todos los factores que conforman ese contexto, los cuales son:

el plan de estudios, la infraestructura de la escuela, el perfil del alumno, la metodología didáctica, el proceso mismo de enseñanza-aprendizaje, la filosofía de la institución, recursos materiales y humanos, etc. deben estar estructurados y correlacionados de tal forma que todos en conjunto trabajen para lograr una mejor preparación de los alumnos que egresarán de esa institución, en este caso en el nivel superior, que es el que nos atañe directamente.

Este trabajo en conjunto corresponde a la llamada planeación educativa, mediante la cual se pretende solucionar los problemas educativos a los que se enfrentan las instituciones en la actualidad, referentes a la preparación de alumnos, procurando que sean competentes en el mercado y obviamente útiles a las demandas de nuestra cambiante sociedad. Una herramienta mediante la cual la planeación educativa logra su objetivo es el correcto diseño curricular y su permanente evaluación. El currículo engloba a todos los elementos del contexto que involucran al maestro, alumno e institución, que mencionamos con anterioridad.

El currículo de una institución de nivel medio superior con bachillerato técnico puede tener un plan de estudios ordenado por materias; ya sea a nivel horizontal o vertical (Díaz Barriga F. 1990) las cuales deben de tener una secuenciación, que facilite la integración de los conceptos aprendidos en los primeros años, para así relacionarlos con los conocimientos de materias de años superiores; de tal forma que esto facilite el proceso de aprendizaje en el alumno y lograr con éxito el perfil del alumno que egresa, es decir 
los contenidos que el alumno debe dominar (el saber), la actitud del alumno (el ser) y las habilidades necesarias (el saber hacer). Es decir competencias.

La integración de estos conceptos, es decir el aprendizaje en el alumno, se puede definir de varias maneras: mecanicista, o por repetición esto sin que el alumno realice una actividad cerebral más compleja, solo memorística, seguramente pronto olvidará los conceptos adquiridos en esa manera, (esto es la relación o asociación de ideas es muy diferente al proceso memorístico).

En cuanto a las condiciones que supone el modelo propuesto, estas son la participación activa del alumno: la mediación, la guía y el monitoreo del aprendizaje por parte del profesor; el aprovechamiento de los errores y la retroalimentación como fuentes de aprendizaje y la secuenciación de la enseñanza para la obtención del meta conocimiento (Estévez 2002)

El requerimiento de que los alumnos obtengan sus logros individualmente y solo a través de sus propios esfuerzos es manejarnos bajo el paradigma de la teoría del aprendizaje logrado por competencias (Barr 1995)

Las materias como análisis cuantitativo del currículo del Técnico en Laboratorio clínico, tiene en común que durante el proceso utiliza diferentes métodos para analizar una muestra y cuantificar sus componentes, por lo cual son cursos teórico-prácticos por lo cual se pretende que los alumnos puedan integrar dichos contenidos teóricos y prácticos para finalmente obtener el resultado del análisis de la muestra problema sin error.

\section{PLANTEAMIENTO DEL PROBLEMA}

\subsection{Planteamiento del tema de investigación}

El programa por competencias se implementó en enero del 2008 en el bachillerato Técnico de Laboratorio Clínico, siendo en enero del 2010 el primer grupo de la UA de análisis Cuantitativo en donde se aplicó la estrategia didáctica de laboratorio con prácticas secuenciales.

En el presente trabajo su objetivo principal fue establecer diferencia entre los grupos que no llevaron la UA con competencias versus los que si las llevaron obviamente demostrando que son mejores sus promedios de calificaciones. Para en lo sucesivo continuar con el modelo por competencias. Para la aplicación de dicho modelo por competencias se implementó la sesión práctica con una secuencia lógica, la cual se pretende sea una propuesta para otros grupos quienes lleven esta UA 
El Laboratorio con prácticas secuenciales como un espacio para propiciar el aprendizaje significativo en los estudiantes de la preparatoria técnica medica de la UANL en la asignatura de análisis cuantitativo en el modelo por competencias.

Demostrar que el establecer una actividad integradora que se realice en la materia de Análisis Cuantitativo lo cual permitirá que el alumno adquiera la competencia de apropiarse de los conocimientos, habilidades y actitudes de la sesión teórica para que los aplique y transfiera a la sesión práctica.

Para comprobar si existe diferencia significativa entre los promedios y porciento de aprobados en los grupos quienes llevaron el curso por competencias versus los grupos que no llevaron el curso con este modelo educativo. Se registraron calificaciones de tres grupos sin competencias y se compararon con 17 grupos quienes si llevaron el modelo educativo por competencias

\subsection{Objetivos de la investigación}

- Demostrar que el modelo educativo por competencias es mejor que el modelo anterior por objetivos

- Propuesta El laboratorio practico como una actividad ponderable que acremente el aprendizaje significativo por competencias

\subsection{Preguntas de investigación}

- ¿Existe diferencia significativa entre los promedios de calificaciones y el porciento de aprobados de los grupos sin competencias versus los que llevaron la UA con competencias?

- ¿El laboratorio práctico será una actividad ponderable que acrescente el aprendizaje significativo por competencias?

\subsection{Justificación de la investigación}

Eliminar la subjetividad de la evaluación en la unidad de aprendizaje de Análisis Cuantitativo a través de prácticas secuenciales que fomenten el aprendizaje significativo en el modelo por competencias, demostrando que es mejor el sistema por competencias que el modelo educativo anterior por objetivos

\subsection{Viabilidad de la investigación}

- Recursos materiales: Concentrado de calificaciones de la Unidad de aprendizaje de análisis cuantitativo de las generaciones comprendidas de enero del 2007 a enero del 2017 
- Encuesta aplicada en línea a una población de 100 exalumnos sobre el laboratorio práctico de dicha UA

- Recursos humanos. Colaboración de exalumnos quienes cursaron la UA de análisis Cuantitativo en el período 2007 al 20017

- Recursos financieros : hojas y cartucho de impresora

\section{Hipótesis}

- Si existe diferencia significativa entre los promedios y porciento de aprobados entre los grupos quienes llevaron el modelo educativo por competencias y los que no los llevaron

- No existe diferencia significativa entre los promedios y porciento de aprobados entre los grupos quienes llevaron el modelo educativo por competencias y los que no los llevaron

\section{MARCO TEORICO}

\subsection{Enseñanza basada en competencias}

Relevancia y pertinencia -en el MCC de la RIEMS- del diseño de la estrategia didáctica. Actualmente la educación en el Nivel Medio Superior en México, demanda buscar nuevas estrategias para promover el desarrollo de competencias en los estudiantes y estar acorde con los lineamientos del SNB. Nuestro campo de acción es en el bachillerato Técnico de Laboratorio Clínico que es impartido en la Escuela y Preparatoria Técnica Medica de la Universidad Autónoma de Nuevo León.

En esta dependencia se observa la siguiente problemática: Es conocido que el alumno cuando carece de las competencias necesarias para aprobar la materia de análisis Cualitativo, carece del conocimiento, habilidades y actitudes para cursar la asignatura de análisis Cuantitativo; por eso si no la aprueba en segunda oportunidad se queda en tercera y esto le impide cursar la materia subsecuente (A. Cuantitativo).

De tal manera que esto trae más prejuicio que beneficio en el alumno ya que al no poder comprender los nuevos conceptos en la materia superior, el alumno también la reprueba. Es lamentable ver como hay alumnos que "sueñan" con poder llevar las materias superiores sin haber aprobado y obviamente sin haber aprendido los conceptos que le son básicos para lograr el aprendizaje significativo; en otras palabras demostrar la importancia de que el alumno lleve las materias en forma secuencial, para garantizar un mayor rendimiento del alumno y esto conllevaría a centrar en la realidad al alumno, 
haciéndole ver que es importante que apruebe la materia base antes de cursar la materia secuencial superior, pues esto implica: llevar la secuencia adecuada.

En dichos programas se contemplan las siguientes competencias profesionales y genéricas, para la unidad de aprendizaje de Análisis Cuantitativo:

Para la unidad de aprendizaje de Análisis Cuantitativo las competencias profesionales son:

1. Aplica los conceptos básicos de la química analítica para la identificación y cuantificación de componentes presentes en una muestra, mediante la realización de prácticas de laboratorio

2. Aplica las leyes y propiedades de los conceptos básicos de la química inorgánica

3. Desarrolla habilidades y destrezas necesarias para su desempeño en el área clínica de laboratorio.

La educación media superior en México tiene como desafío responder a las necesidades de cobertura, lo cual incide de manera negativa en la equidad que debe promover el sistema educativo. Adicionalmente, se observa que existen importantes obstáculos para garantizar la calidad de la educación que se imparte en este nivel. Una de las responsabilidades que tiene el Gobierno es de cerciorarse que los jóvenes encuentren oportunidades para realizarse en la cercanía de su vida adulta: considerando que en el 2010 nuestro país alcanzo el máximo histórico en el número de jóvenes entre 16 y 18 años, los cuales constituyen el grupo de edad de cursar la educación Media Superior Las tendencias actuales para el siglo XXI, en relación a la segunda década suponen que el EMS en nuestro país tendrá un rezago de 50 años. Esto implica la necesidad de un esfuerzo conjunto de autoridades educativas docentes, estudiantes, padres de familia y sociedad en general que aspira a mayores niveles de bienestar en una etapa en que el número de jóvenes alcanza su máximo histórico.

El punto de partida para definir la identidad de la Educación Media Superior (EMS) en el país es enfrentar los retos que se plantea. El camino que tome este nivel educativo deberá dar atención a estos retos, los cuales se resumen en los siguientes:

- Ampliación de la cobertura

- Mejoramiento de la calidad

- Búsqueda de la equidad 
En la coyuntura actual resulta esencial fortalecer la cobertura, equidad y calidad en la EMS que permitirá fortalecer las habilidades y conocimientos de los jóvenes para un mejor desarrollo personal, profesional y laboral.

De acuerdo a lo anteriormente planteado la propuesta didáctica está acorde con que la pertinencia y relevancia de los planes de estudio, significa que dichos planes deben de ser compatibles con las competencias y conocimientos obligatorios permitiendo que se den las múltiples y necesarias relaciones entre escuela y entorno. Esta cualidad a la que deben atender los planes de estudio debe de abarcar la pertinencia personal, social y laboral del contexto de las circunstancias del mundo actual considerando que de esta manera podrá incrementarse el nivel de motivación hacia el estudio, y por el contrario, evitar la deserción escolar.

Por mucho tiempo, la enseñanza de las disciplinas se ha organizado en torno a temas. Casi siempre ordenados por su evolución o integración cronológica, o bien a partir de la elección de aquellos que han sido cruciales en la construcción del cuerpo de conocimientos de una ciencia o campo profesional del grupo en el control o liderazgo. La disciplina es un recorte, una delimitación de entre un vasto conjunto de conocimientos, y se decide priorizando de acuerdo a criterios establecidos por alguna instancia académica, en el mejor de los casos.

Los contenidos orientados desde una perspectiva de competencias, deberán tener cierta secuencia en que los estudiantes deberán seguir para dominar procesos o ejecuciones en los que integren conocimientos, habilidades, actitudes y valores.

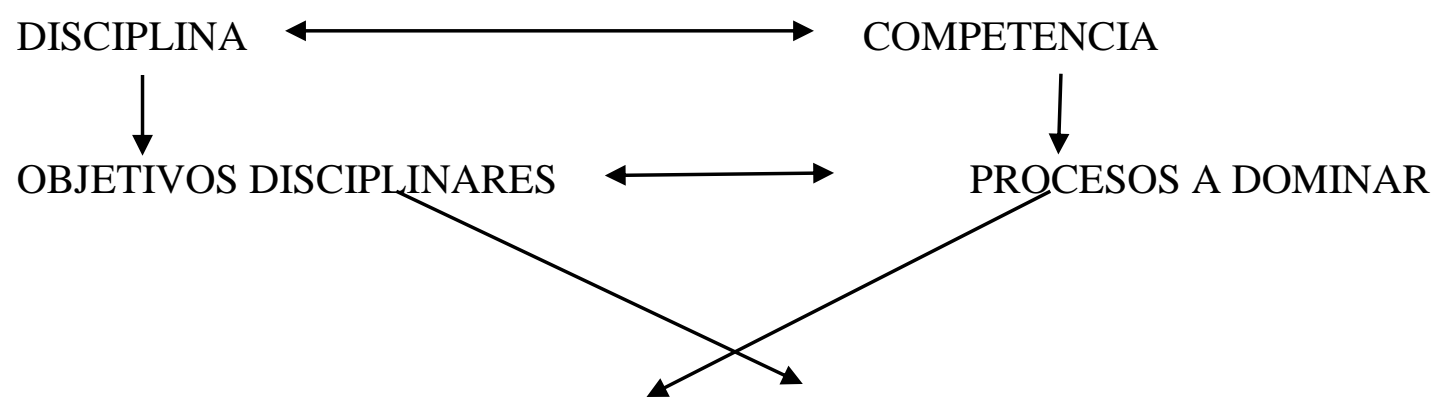

COMPETENCIA INTEGRADORA

(Productos de aprendizaje) 


\section{Competencias}

Domina y estructura los contenidos de las asignaturas a su cargo para desarrollar las secuencias que faciliten el aprendizaje

Por lo cual se determina los productos de aprendizaje por competencias en este caso actividad integradora.

La estrategia didáctica se fundamenta en el enfoque pedagógico del constructivismo y la teoría Ausubel (1989) que señala la forma en que el alumno adquiere el conocimiento nuevo relacionándolo con lo que ya sabe, lo integra a la estructura cognitiva de su cerebro en forma ordenada, con cierta secuencia, es decir, si un concepto es general, por ejemplo: los mamíferos son animales de sangre caliente y se alimentan con leche, posteriormente el alumno recibe la información más particular de que el gato es un mamífero, entonces por un proceso cognitivo deduce que el gato toma leche y tiene sangre caliente, estableciéndose una estructura cognitiva en su cerebro, si el alumno no contara con ese concepto general, carecería de significado para él, el hecho de que el gato sea un mamífero.

De tal forma un currículo de cualquier bachillerato debe de estar diseñado de manera, que las materias del plan de estudios cuenten con cierta secuencia (Díaz B.F,. 1990); (la relación entre el aprendizaje significativo y el currículo está dada por la teoría de la asimilación (Ausubel 1989);para que el alumno realice el llamado aprendizaje significativo es necesario además que adquiera los conceptos generales y básicos es las primeras materias, para que posteriormente vaya integrando los nuevos conocimientos de las materias subsecuentes; sin dejar de mencionar que el contenido temático de las materias base sea general, y el contenido de las materias secuenciales sea más específico. Para evaluar de manera completa al alumno se diseñó para cada curso una actividad integradora la cual consiste en lo siguiente:

El alumno retomara los conceptos aprendidos en sus cursos pasados como lo son: Química I y II, Matemáticas, y de Análisis cualitativo como materias previas para la UA de Análisis Cuantitativo para el Técnico en Laboratorio clínico.

\subsection{Prácticas de laboratorio como herramienta didáctica}

Como lo menciona Manjarrez (2017) “Las competencias científicas están íntimamente relacionadas con las prácticas de laboratorio de que aporta el pensamiento basado en Sistema, desarrolla la capacidad para la toma de decisiones y la utilización de la ciencia" 
así como se desarrollan experiencias grupales, donde se potencia la actividad, la confianza y el respeto en los equipos de trabajo Ayala-Valenzuela (2007)

Dichas prácticas con una secuencia lógica en la aplicación de conceptos (anclajes) para lograr un buen aprendizaje, asociación y retención de los nuevos conceptos Cardona (2013) además que le permiten a los estudiantes analizar, sintetizar y evaluar la información, no simplemente actuar como espectadores en el laboratorio Durango (2015) en el caso de que sean prácticas demostrativas como pasa en algunos casos.

Espinosa-Ríos (2016) Concluyen en su estudio que las prácticas de laboratorio son concebidas como una estrategia didáctica para la enseñanza -aprendizaje de reacciones químicas, permitió el Desarrollo de algunas habilidades científicas y un aprendizaje significativo de los conceptos.

\section{MARCO METODOLOGICO}

\subsection{Diseño argumentado y comunicable de la estrategia didáctica propuesta.}

\subsubsection{Fundamentación}

En el plan de estudios de dicho bachillerato las materias del área de química analítica se imparten en el tercer y cuarto semestre:

- Análisis Cualitativo 3er semestre

- Análisis Cuantitativo 4to semestre

Cuando se imparten las materias curriculares bajo la estrategia didáctica por competencias, además de que se pretende que el alumno aprenda a aprender (Estévez 2002) y que tenga ciertas actitudes y habilidades para lograr el aprendizaje significativo (Ausbel, 1989), también es necesario establecer diferentes estrategias para poder evaluar pedagógicamente el desempeño del alumno.

El modelo didáctico que aquí se presenta permite diseñar la enseñanza como base del uso de estrategias cognitivas (Estévez 2002)

En cuanto a las condiciones que supone el modelo, estas son la participación activa del alumno: la mediación, la guía y el monitoreo del aprendizaje por parte del profesor; el aprovechamiento de los errores y la retroalimentación como fuentes de aprendizaje y la secuenciación de la enseñanza para la obtención del meta conocimiento (Estévez 2002) El requerimiento de que los alumnos obtengan sus logros individualmente y solo a través de sus propios esfuerzos es manejarnos bajo el paradigma de la teoría del aprendizaje logrado por competencias (Barr 1995) 
Las materias como análisis cuantitativo del currículo del Técnico en Laboratorio clínico, tiene en común que durante el proceso utiliza diferentes métodos para analizar una muestra y cuantificar sus componentes, por lo cual son cursos teórico /prácticos que permite el desarrollo de competencias.

\subsubsection{Diseño de la Estrategia Didáctica}

En seguida se detalla pormenorizadamente la estrategia didáctica:

\begin{tabular}{|c|c|}
\hline \multicolumn{2}{|c|}{ Nombre de la Unidad de aprendizaje: Análisis Cuantitativo } \\
\hline \multicolumn{2}{|c|}{ Semestre en que aplica las estrategia didáctica: Cuarto } \\
\hline Grupo: 406 & Número de alumnos: 65 \\
\hline \multicolumn{2}{|c|}{$\begin{array}{l}\text { Nombre de la estrategia didáctica: El Laboratorio Clínico como un espacio para propiciar el } \\
\text { aprendizaje significativo en los estudiantes de la preparatoria técnica medica de la UANL en } \\
\text { la asignatura de análisis cuantitativo (Actividad Integradora) }\end{array}$} \\
\hline Contexto: Laboratorio & Duración de la estrategia didáctica: 1 semestre \\
\hline Propósito y objetivos a desarrollar: & $\begin{array}{l}\text { - Aplica los conceptos adquiridos en química I y II } \\
\text { sobre la nomenclatura química de Ácidos y bases } \\
\text { así como los conceptos de concentración Normal } \\
\text { que son retomados de Análisis Cualitativos } \\
\text { - Evalúa su propio desempeño en la elaboración } \\
\text { correcta del reactivo en la semana anterior, } \\
\text { obteniendo el valor de la normalidad real y que } \\
\text { tanta desviación tiene del valor requerido. } \\
\text { - Comprende la importancia de estandarizar la } \\
\text { solución de Na OH para poder utilizarla como } \\
\text { estándar secundario y así calcular el \% de Ac } \\
\text { acético presente en la muestra de vinagre que trajo } \\
\text { al laboratorio } \\
\text { Comprende la importancia de estandarizar la } \\
\text { solución de HCl para poder utilizarla como } \\
\text { estándar secundario y así calcular el \% de } \\
\text { bicarbonato presente en la muestra de un antiácido } \\
\text { (sal de uvas) que trajo al laboratorio } \\
\text { Juzga su trabajo y reconoce sus errores en caso de } \\
\text { obtener un porcentaje elevado, por lo cual tiene la } \\
\text { oportunidad de estandarizar nuevamente las } \\
\text { soluciones o en su caso volver a realizar un } \\
\text { reactivo en el que se tenga duda, }\end{array}$ \\
\hline Competencias disciplinares & Competencias genéricas \\
\hline $\begin{array}{l}\text { Aplica los conceptos básicos de la } \\
\text { química analítica para la } \\
\text { identificación y cuantificación de } \\
\text { componentes presentes en una } \\
\text { muestra, mediante la realización de } \\
\text { prácticas de laboratorio }\end{array}$ & $\begin{array}{l}\text { - Escucha, interpreta y emite mensajes pertinentes } \\
\text { en distintos contextos, mediante la utilización de } \\
\text { medios, códigos y herramientas apropiados. } \\
\text { - Desarrolla innovaciones y propone soluciones a } \\
\text { problemas a partir de métodos establecidos. } \\
\text { - Sustenta una postura personal sobre temas de interés } \\
\text { y relevancia general, considerando otros puntos de } \\
\text { vista de manera crítica y reflexiva. }\end{array}$ \\
\hline
\end{tabular}




\begin{tabular}{|c|c|}
\hline $\begin{array}{l}\text { 1. Aplica las leyes y propiedades de } \\
\text { los conceptos básicos de la } \\
\text { química inorgánica } \\
\text { 2. Desarrolla habilidades y } \\
\text { destrezas necesarias para su } \\
\text { desempeño en el área clínica de } \\
\text { laboratorio. }\end{array}$ & $\begin{array}{l}\text { Participa y colabora de manera efectiva en equipos } \\
\text { diversos }\end{array}$ \\
\hline \multicolumn{2}{|l|}{ Evidencias de aprendizaje } \\
\hline \multicolumn{2}{|c|}{$\begin{array}{l}\text { 1. Una bitácora en un diario donde se especifique el fundamento, objetivo, material } \\
\text { procedimiento, dibujos resultados y conclusiones de cada sesión } \\
\text { 2. Portafolio de reportes de prácticas con el mismo contenido del diario, realizados en } \\
\text { computadora. } \\
\text { 3. El reporte que entregara el día del examen práctico cuya calificación estará en función } \\
\text { del \% de error obtenido en la cuantificación de analitos. }\end{array}$} \\
\hline
\end{tabular}

En seguida se describen las actividades de aprendizaje que se desarrollaron

Actividad integradora Análisis cuantitativo. Enfocada al método volumetría para cuantificación de analitos,

Metodología: Durante todo el semestre se realizaran prácticas de laboratorio una sesión de una hora por semana, siendo 7 semanas las que se ocuparían.

\section{Actividad de aprendizaje 1.}

Semana 1: Preparación de soluciones: $\mathrm{NaOH} 0.1 \mathrm{~N}$ y HCl 0.1N 1000mL

Competencia de aprendizaje: Aplica los conceptos adquiridos en química I y II sobre la nomenclatura química de Ácidos y bases así como los conceptos de concentración Normal que son retomados de Análisis Cualitativos.

Procedimiento: El alumno realiza los cálculos necesarios para preparar las soluciones del ácido y del hidróxido.

\section{Actividad del maestro:}

- Corrobora que los cálculos estén correctos

- Revisa que la afloración este correcta.

- Dichas soluciones son guardadas en botellas de plástico limpias de agua (1 lto) debidamente identificadas

\section{Actividad de aprendizaje 2 .}

Semana 2: Preparación de soluciones: Na2CO3 0.1 N Biftalato de potasio 0.1N 100mL Competencia de aprendizaje: Aplica los conceptos adquiridos en química I y II sobre la nomenclatura química sales así como los conceptos de concentración Normal que son retomados de Análisis Cualitativos. 


\section{Procedimiento:}

- El alumno realiza los cálculos necesarios para preparar las soluciones de del $\mathrm{Na}_{2} \mathrm{CO}_{3}$ y del biftalato de potasio

\section{Actividad del maestro:}

- Corrobora que los cálculos estén correctos

- Revisa que la afloración este correcta

- Dichas soluciones son guardadas en botellas de plástico limpias de agua (500 mL) debidamente identificadas

\section{Actividad de aprendizaje 3}

Semana 3: Estandarización del $\mathrm{NaOH}$

Competencia de aprendizaje: Evalúa su propio desempeño en la elaboración correcta del reactivo en la semana anterior, obteniendo el valor de la normalidad real y que tanta desviación tiene del valor requerido.

\section{Procedimiento:}

- El alumno realiza la estandarización del $\mathrm{NaOH}$ utilizando una alícuota de $10 \mathrm{~mL}$ del biftalato y el indicador fenolftaleína, el vire es de incoloro a rosa.

\section{Actividad del maestro:}

- Supervisa que el alumno mida correctamente las alícuotas, de que afore bien la bureta y de que llegue al punto de vire sin haberse pasado.

\section{Actividad de aprendizaje 4}

Semana 4: Estandarización del $\mathrm{HCl}$

Competencia de aprendizaje: Evalúa su propio desempeño en la elaboración correcta del reactivo en la semana anterior, obteniendo el valor de la normalidad real y que tanta desviación tiene del valor requerido.

\section{Procedimiento:}

- El alumno realiza la estandarización del $\mathrm{HCl}$ utilizando una alícuota de $10 \mathrm{~mL}$ del $\mathrm{Na} 2 \mathrm{CO} 3$

- Y el indicador naranja de metilo, el vire es de amarillo a durazno.

\section{Actividad del maestro:}

- Supervisa que el alumno mida correctamente las alícuotas, de que afore bien la bureta y de que llegue al punto de vire sin haberse pasado.

\section{Actividad de aprendizaje 5}


Semana 5. Determinación de la acidez del vinagre (\% Ac acético)

Competencia de aprendizaje: Comprende la importancia de estandarizar la solución de $\mathrm{NaOH}$ para poder utilizarla como estándar secundario y así calcular el \% de Ac acético presente en la muestra de vinagre que trajo al laboratorio

\section{Procedimiento:}

- Afora la bureta de $50 \mathrm{ml}$ con la solución estándar de $\mathrm{NaOH}$, mide 3 alícuotas de la solución de vinagre y le agrega el indicador fenolftaleína, posteriormente observa el punto de vire: de incoloro a rosa fusa, realiza cálculos para la obtención del porcentaje de ac acético presente.

\section{Actividad del maestro:}

- Supervisa que el alumno mida correctamente las alícuotas, de que afore bien la bureta y de que llegue al punto de vire sin haberse pasado. Y junto con el calculan él \% de analitos y comparándolo con el valor comercial del vinagre y esto lo hace consciente del \% error registrado.

\section{Actividad de aprendizaje 6}

Semana 6. Determinación de Bicarbonato de sodio en un antiácido

Competencia de aprendizaje: Comprende la importancia de estandarizar la solución de $\mathrm{HCl}$ para poder utilizarla como estándar secundario y así calcular el \% de bicarbonato presente en la muestra de un antiácido (sal de uvas) que trajo al laboratorio

\section{Procedimiento:}

- Afora la bureta de $50 \mathrm{ml}$ con la solución estándar de $\mathrm{HCl}$, mide 3 alícuotas de la solución de sal de uvas y le agrega el indicador naranja de metilo, posteriormente observa el punto de vire: de naranja a melón, realiza cálculos para la obtención del porcentaje de bicarbonato de sodio presente.

\section{Actividad del maestro:}

- Supervisa que el alumno mida correctamente las alícuotas, de que afore bien la bureta y de que llegue al punto de vire sin haberse pasado. Y junto con el calculan él \% de analitos y comparándolo con el valor comercial del bicarbonato en la sal de uvas y esto lo hace consciente del \% error registrado.

\section{Actividad de aprendizaje 7}

Semana 7: Tiempo de enmendar errores 
Competencia de aprendizaje: Juzga su trabajo y reconoce sus errores en caso de obtener un porcentaje elevado, por lo cual tiene la oportunidad de estandarizar nuevamente las soluciones o en su caso volver a realizar un reactivo en el que se tenga duda,

\section{Procedimiento:}

- Solo si el alumno lo desea realizara de nuevo la estandarización de las soluciones estándares de $\mathrm{NaOH}$ y de $\mathrm{HCl}$, con el procedimiento descrito anteriormente en las actividades 3 y 4 .

\section{Actividad del maestro:}

- Está presente y ayuda al alumno a detectar el o los errores, y solo a petición del alumno se dará oportunidad de realizar nuevamente el procedimiento a corregir. Si el alumno considera que todo es correcto entonces no realiza esta práctica.

- Finalmente en la semana 8 se realiza un examen final en donde se le proporciona una muestra de un ácido o una base y el alumno deberá de reportar:

$\checkmark$ El titulante que utilizara

$\checkmark$ El indicador

$\checkmark$ El \% de analitos determinado.

\section{Evidencias de aprendizaje}

- Una bitácora en un diario donde se especifique el fundamento, competencia, material procedimiento, dibujos resultados y conclusiones de cada sesión

- Portafolio de reportes de prácticas con el mismo contenido del diario, realizados en computadora.

- El reporte que entregara el día del examen práctico cuya calificación estará en función del \% de error obtenido en la cuantificación de analitos.

\section{Evaluación}

Se utiliza una rúbrica para evaluar el trabajo en equipo, las cuales ayudaran a los alumnos y al maestro a supervisar y criticar su propio trabajo, coadyuvan a eliminar la subjetividad en la evaluación y en la ubicación por niveles de los alumnos (Díaz-Barriga 2002) p 390

\section{Ejemplo: Rubrica para trabajo en equipo}

\section{Figura. 1}

\begin{tabular}{|c|c|c|c|c|c|}
\hline Atributos & Excelente & Muy Bien & Bien & Satisfactorio & $\begin{array}{c}\text { No } \\
\text { Satisfactorio }\end{array}$ \\
\hline $\begin{array}{l}\text { Mantienen el orden y } \\
\text { disciplina en el equipo }\end{array}$ & & & & & \\
\hline
\end{tabular}




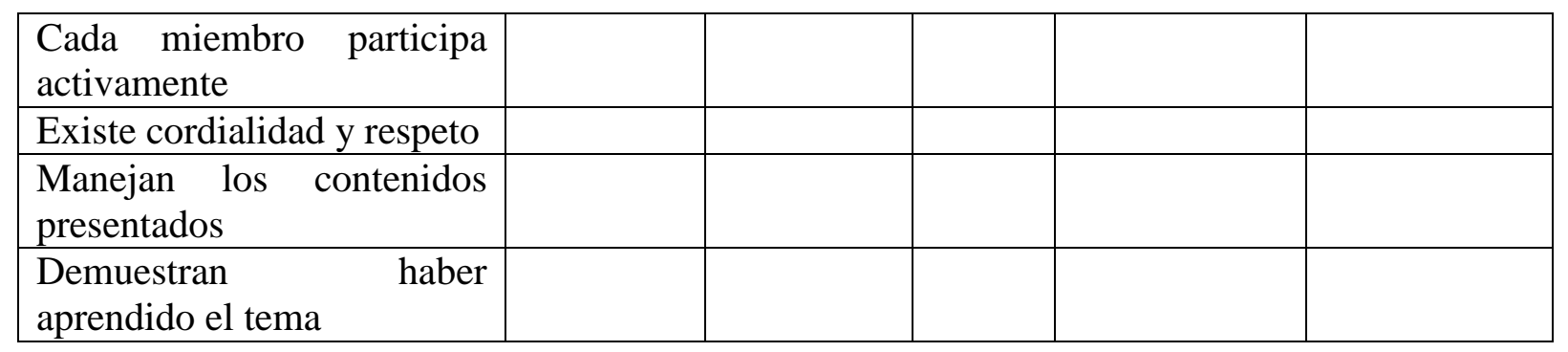

A continuación se argumenta y justifica como la estrategia didáctica propuesta es coherente con las competencias, el o los propósito (s), los contenidos y los productos.

La importancia de generar el aprendizaje en el alumno es el por qué y para qué de nuestra labor docente. El aprendizaje por competencias logra la integración total de las tres áreas básicas de todo ser humano: lo cognitivo, afectivo y psicomotor, en especial para los alumnos de un bachillerato técnico, fusionar estos aspectos logra que sea competente para trabajar con pacientes, (actitud), saber cómo tomar las muestras (aptitud) axial como conocer los diferentes fundamentos científicos para las diversas determinaciones de los analitos de interés en un análisis clínico.

En seguida se justifica y fundamenta como la estrategia didáctica a través de los productos desarrolla las competencias y se alcanza el propósito.

Cuando se utiliza el enfoque profundo para realizar una tarea como es el caso de este diseño didáctico, los estudiantes tienen sentimientos positivos: interés, sentido de la importancia, sensación de desafío e incluso euforia. Aprender es un placer (Biggs 2005) Está demostrado que si el alumno cuando adquiere un ambiente positivo de aprendizaje, se tiene actitudes y percepciones positivas lo cual lleva a que el alumno aprenda más y este será más fácil.

Cuando los alumnos sienten la necesidad de saber, procuran centrarse en el significado subyacente: en ideas principales, temas principios o aplicaciones satisfactorias (Biggs 2005)

Esto comunica cuando los alumnos que aprenden extienden y reafirman el conocimiento, siguen adquiriendo conocimiento dándole un uso significativo para ellos, y hace que extiendan más aun el gusto por aprender más. (Marzano 2005)

Pues bien el que el alumno juzgue finalmente su labor durante el semestre y en caso de haber cometido errores, detectarlos y modificarlos, implica que se cumplieron las expectativas, es decir las competencias tanto disciplinares como generales. 


\subsection{Material y métodos}

Material: Paquete estadístico SPSS 22

Metodología: Durante todo el semestre se realizaron prácticas de laboratorio una sesión de una hora por semana, siendo 7 semanas las que se ocuparon.

Se estableció una comparación de rendimiento académico de 20 grupos de análisis cuantitativo en el cual se implementó esta actividad integradora contra dos grupos los cuales no llevaron esta actividad ni el programa por competencias, esto es del $2007 \mathrm{y}$ 2008 que no llevaron competencias contra los grupos desde el 2009 al 2017 quienes llevaron el laboratorio secuencial en su programa por competencias

Se realizó una encuesta en línea con una población de ex alumnos quienes cursaron la UA de Análisis Cuantitativo en el período de 2007 al 2017, con 10 preguntas relacionadas con el laboratorio de prácticas

\section{ANALISIS Y RESULTADOS}

Se capturaron las calificaciones finales de los grupos quienes llevaron la UA de Análisis Cuantitativo sin competencias, siendo tres y las demás calificaciones de los grupos con competencias siendo 17.

Se observó lo siguiente:

Aplicando el paquete estadístico SSPS 22 y realizando la comparación de los tres grupos vs los 17 obteniendo los promedios por grupo y aplicando la prueba Chi cuadrada para observar si existe diferencia significativa entre estos valores (tabla 1)

\begin{tabular}{|c|c|c|c|c|c|}
\hline Grupos & Numero & Mínimo & Máximo & Media & $\begin{array}{c}\text { Desviación } \\
\text { estándar }\end{array}$ \\
\hline 2007 & 17 & 30 & 88 & 65 & 20.37 \\
\hline 2008 & 52 & 10 & 98 & 68.98 & 22.24 \\
\hline 2009407 & 64 & 11.90 & 97.60 & 72.81 & 16.71 \\
\hline 2009408 & 29 & 57.20 & 93.90 & 73.53 & 9.79 \\
\hline 2010407 & 29 & 54.30 & 95 & 76.1 & 10.55 \\
\hline 2010408 & 33 & 54 & 96 & 82.27 & 8.95 \\
\hline 2011407 & 28 & 53 & 95 & 76.92 & 8.02 \\
\hline 2011408 & 25 & 18 & 94 & 72.72 & 24.15 \\
\hline 2012407 & 25 & 29 & 91 & 75.2 & 12.41 \\
\hline 2012408 & 28 & 59 & 94 & 82.14 & 7.75 \\
\hline 2013407 & 30 & 17 & 94 & 77.13 & 16.9 \\
\hline $2013 \quad 408$ & 23 & 17 & 81 & 65.47 & 17.96 \\
\hline $2014 \quad 407$ & 32 & 9.8 & 96.20 & 86.44 & 14.95 \\
\hline $2014 \quad 408$ & 23 & 47 & 86 & 77.46 & 8.21 \\
\hline $2015 \quad 407$ & 38 & 10 & 94 & 82.86 & 13.33 \\
\hline $2015 \quad 408$ & 27 & 38 & 95 & 74.92 & 14.63 \\
\hline
\end{tabular}




\begin{tabular}{|cc|c|c|c|c|c|}
\hline 2016 & 407 & 29 & 50 & 94 & 81.03 & 9.67 \\
\hline 2016 & 408 & 17 & 30 & 88 & 66.76 & 20.37 \\
\hline 2017 & 407 & 35 & 31 & 95 & 80.25 & 12.82 \\
\hline 2017 & 408 & 41 & 30 & 91 & 74.41 & 14.25 \\
\hline
\end{tabular}

Se observó diferencia significativa entre los grupos del 2007, 2008 y 2009 vs los del 2010, 2011,2012(grupo 407), 2013,2014, 2015,2016 y 2017(grupo 407) siendo mayor los promedios en los grupos que llevaron las competencias (valores menores a 0.05 )

Solo en dos grupos en el 2013 y2016 (grupo 408) no existe diferencia significativa entre sus promedios y los promedios de los grupos sin competencias, muy seguramente ya que los grupos con terminación 08 corresponden a los alumnos quienes no eligieron como primera opción esa técnica (Laboratorio Clínico) y pues no tuvieron más remedio que elegir esa técnica.

Con relación a la encuesta aplicada en línea a 100 exalumnos quienes llevaron el curso de Análisis Cuantitativo durante el período de 2007 al 2017 estos son los resultados.

Se aplicó el análisis de fiabilidad encontrándose un índice alfa que Kronbach de 0.415 que significa una relación moderada entre variables.

Como se logra apreciar en un $96 \%$ los alumnos contestaron para la pregunta 1 que es muy necesario que la materia contara con laboratorio de prácticas.

Para la pregunta dos un $72 \%$ consideró que si era muy importante el orden como se llevaron las prácticas

En la pregunta 3 el 79\% señaló como muy importante que se cuente con un diario para escribir el fundamento y resultados en la práctica.

Observando la pregunta 4 un $72 \%$ opinó como muy aplicativas las sesiones prácticas de laboratorio

Para la pregunta 5 el porcentaje observado sobre la importancia de corregir errores en reactivos y procedimientos fue de un $91 \%$

En la pregunta 6 en donde se les cuestiona sobre su desempeño en trabajo por equipo el $50 \%$ manifiesta que si tiene un mejor desempeño colaborando con su compañero, el $42 \%$ señala que es un buen desempeño( no muy deseable) y el $7 \%$ manifiesta que tiene poco desempeño haciendo la práctica en equipo

Observando la pregunta 7 en donde se les pide que señalen si es útil corregir errores de los reportes escritos para entregarlos al final un $73 \%$ manifiesta que es muy importante, un $20 \%$ que es importante, un $5 \%$ poco importante y un $2 \%$ nada importante. 
Como se logra apreciar en la pregunta 8 sobre si están conscientes de que el error en preparar reactivos les afecta a su examen práctico final el $49.5 \%$ de los alumnos contestó que si existe mucha relación, $49.5 \%$ manifiesta que si existe relación y el 1\% restante opina que no existe relación.

En la pregunta 9 sobre el ambiente de trabajo en la practica $84 \%$ opinó que era muy positivo, el $14 \%$ positivo y $2 \%$ poco positivo

En la pregunta 10 se observa que la población encuestada 7 alumnos de 100 no llevaron el curso por competencias y 93 alumnos encuestados si llevaron el curso por competencias

\section{CONCLUSIONES}

Generar el aprendizaje en el alumno es el por qué y el para qué de la labor docente. El aprendizaje por competencias logra la integración total de las tres áreas básicas de todo ser humano: lo afectivo, lo cognitivo y lo psicomotor (Argudín 2005), en especial para los alumnos de un bachillerato técnico; fusionar estos aspectos logra que sea competente para trabajar con pacientes (actitud), saber cómo tomar las muestras (aptitud) axial como conocer los diferentes fundamentos científicos para las diversas determinaciones de los analitos de interés en un análisis clínico. Una competencia está muy alejada de lo que se considera un aprendizaje mecánico e implica un mayor grado de significatividad y funcionalidad posible, ya que para poder ser utilizada deben tener sentido tanto la propia competencia como sus componentes procedimentales, actitudinales y conceptuales (Zavala 2008)

Con los resultados de aprovechamiento (promedios por grupo y valores altos de calificación) del curso de Análisis Cuantitativo con el modelo educativo por competencias observamos que si existe diferencia significativa con valores mayores en estos grupos contra los grupos que no llevaron dicho modelo. Es decir se puede afirmar que el modelo por competencias aplicado desde el 2010 para los grupos de Análisis Cuantitativo supera al modelo sin competencias.

Con relación a la encuesta, es satisfactorio observar que en un gran porcentaje los alumnos manifestaron estar conscientes de la importancia del laboratorio de prácticas, de su secuencia, metodología, logística y auto evaluación (corregir errores). Solo en el trabajo en equipo si hubo un poco de discrepancia ya que en un porcentaje del $7 \%$ expresan que tuvieron POCO DESEMPEÑO, punto a considerar ya que en cualquier trabajo siempre se deberá de trabajar en equipo, aunque en algunas ocasiones no les agrade. 
Conocer el grado de dominio del alumnado ha adquirido de una o varias competencias sobre todo en la parte práctica de laboratorio es una labor continua la cual se realiza en cada período y se realizaron actualizaciones tanto en la planta docente como en los programas.

Cuando se utiliza el enfoque profundo para realizar una tarea como es el caso de este diseño didáctico, los estudiantes tienen sentimientos positivos: interés, sentido de la importancia, sensación de desafío e incluso euforia. Aprender es un placer (Biggs 2005) Está demostrado que si el alumno cuando adquiere un ambiente positivo de aprendizaje, se tiene actitudes y percepciones positivas lo cual lleva a que el alumno aprenda más y este será más fácil.

Cuando los alumnos sienten la necesidad de saber, procuran centrarse en el significado subyacente: en ideas principales, temas principios o aplicaciones satisfactorias (Biggs 2005)

Esto comunica cuando los alumnos que aprenden extienden y reafirman el conocimiento, siguen adquiriendo conocimiento dándole un uso significativo para ellos, y hace que extiendan más aun el gusto por aprender más. (Marzano 2005)

\section{Recomendaciones}

A continuación se abordarán cuatro puntos que consideramos de suma importancia que se lleven a cabo:

1. Involucrar a todos los integrantes de la institución en la interpretación y revisión curricular. Lo anterior se refiere a que no solamente el círculo administrativo de la institución tome decisiones o evalúe lo que corresponde al currículo, los maestros, el personal profesional de apoyo y por qué no los alumnos. E informarlos sobre lo que es la REMS el MCC y el cambio de los planes y programas por competencias.

2. Contar con expertos en diseño curricular que asesoren a las instituciones de educación superior en la aplicación del plan de estudios. En este punto recordemos que el trabajo de la planeación educativa y del diseño curricular es una labor multidisciplinaria, por lo cual debería de existir apertura y madurez en los directivos de las instituciones, con el motivo de aceptar la ayuda en esta labor tan importante.

3. Que los maestros revisen la organización de sus programas en función del currículo vigente, basado en competencias para revisar su congruencia y pedir asesoría para realizar las mejoras. Muchos programas son obsoletos y así como la demanda de la 
sociedad es cambiante, también el perfil del egresado, por lo tanto el currículo debe de estar en continua revisión y evaluación y desde luego los programas de las materias de la carrera para que exista congruencia en el proceso.

4. Proponer que los maestros conozcan y adapten metodologías y estrategias para lograr el aprendizaje significativo. Para realizar lo anterior es recomendable capacitar al personal docente sobre los tipos de aprendizaje que existen, así como comprender la importancia del aprendizaje significativo en los alumnos, seguido a esto la utilización de diversas dinámicas o aplicación de la didáctica crítica para llevarlo a cabo.

\section{REFERENCIAS BIBLIOGRAFICAS.}

Acuerdo Secretarial 442 Por el que se establece el sistema nacional de bachillerato Competencias docentes y sus respectivos atributos, que expresan el Perfil del Docente de Educación Media Superior (2008)

Acuerdo número 444 (2008) por el que se establecen las competencias que constituyen el marco curricular común del Sistema Nacional de Bachillerato Competencias docentes y sus respectivos atributos, que expresan el Perfil del Docente de Educación Media Superior

Acuerdo Secretarial No. 447 (2008) Competencias docentes y sus respectivos atributos, que expresan el Perfil del Docente de Educación Media Superior

Aguilera Mario, Diseño de planes y programas. (Apuntes de clase) Filosofía y Letras pos grado. Monterrey N.L. Marzo 1995

Apostel, et al., Interdisciplinariedad. Problemas de la enseñanza e investigación en las universidades, pXIII. Revista de la Educación Superior Número 14

Volumen 4 • Abril - Junio de 1975

Argudín Y. Educación basada en competencias. Ed Trillas (2005) 28, 29, 64

7Arnaz, J.A. La planeación curricular, Trillas México, 1996

Arredondo, V.A. " Algunas tendencias predominantes y características de la investigación sobre desarrollo curricular", en Documento Base, Congreso Nacional de Investigación Educativa, vol. I, México, 1981

Ausubel, D.P. The psychology of meaningful verbal learning .New York: Grune\&Stratton 1963

Ausubel, D.P. \& Robinson, F.G. School learning: an introduction to educational psychology. New York: Holt, rinehart \& Witson. 1969. 
Ausubel, David Paul, Psicología educativa un punto de vista cognoscitivo, 2da Ed Trillas 2009

Ayala-Valenzuela Ricardo Educación Médica Superior Didáctica de la enseñanza. Prácticas ejemplares en el sector salud. Vol 21 No 2 Abril-jun 2007 Ciudad de la Habana

Beauchamp, G:A: " Basic components of a curricular theory" , en A.A. Bellack H.M.

Kliebard (dirs) Curriculum and evaluation, Mc Cutchan Publishers Corporation, Berkley, California, 1977

Barr R, Tagg J, Evaluacion Educativa de la enseñanza al aprendizaje un nuevo paradigma para la educación de pregrado CONAEVA 1998

Biggs J, Calidad del aprendizaje universitario NARCEA, SA DE EDICIONES 2005

Burner J.S. Toward, a theory of instruction Cambrige, Mass: Belknap Press of Harvard, University Press.1974

Cardona F Las prácticas de laboratorio como estrategia didáctica Instituto de Educación y Pedagogía Santiago de Cali septiembre 2013

Clases. Enseñanza modular E. de O. de la U.A.B.C. (1976) (pp 16)

Covey Stephen, Los siete hábitos de la gente altamente efectiva, Ed. Paidos, 2015

Craing Mehrens, Psicología educativa contemporánea, concepto, temática y aplicaciones, Ed LIMUSA, 1989

De Leonardo P, La educación superior privada en México, Línea México, 1983.

Díaz Barriga Ángel, Ponencia presentada en el foro nacional de innovaciones en la educación superior, $\quad$ Monterrey N.L., septiembre, 1988.

Díaz-Barriga, F. Lule, et al. "Metodología de diseño curricular para la enseñanza superior", en revista de Perfiles educativos, Díaz-Barriga F. Lule, et al. "Propuesta de organización curricular para la creación de la Licenciatura en psicología educativa", documento inédito, México, 1981.

Díaz Barriga Frida, Metodología de diseño curricular para educación superior, Ed Trillas, reimpresión 2012

Durango P Las prácticas de laboratorio como una estrategia didáctica para desarrollar las competencias básicas en el proceso de enseñanza-aprendizaje de la química Facultad de ciencias Medellín Colombia 2015 
Espinoza-Ríos E Las prácticas de laboratorio; una estrategia didáctica en la construcción del conocimiento científico escolar Red de revistas científicas de américa latina y del caribe, España y Portugal Vol 12 No 1 enero-junio 2016

Estévez E, Enseñar a aprender Estrategias cognoscitivas Paidos reimpresión 2005

Gagne, Robert, La planificación de la enseñanza, sus principios Ed. Trillas. México, 2011.

Glazman, R. e Ibarrola de M, Diseño de planes de estudio, CISE-UNAM, México, 1981. Grass Sociología de la Educación- Narcea S.A. de Ediciones, Madrid 3a Ed,1985.

Guevara, Diseño curricular, (1976) México: UAM-X, DCBS

Johnson, H, Curriculum y educación, Troquel, Buenos Aires, 1970

Johnson, H. "Definitions and models in Curriculum and evaluation, Mc Cutchan Publishers Corporation, Berkley, California, 1977. Pp127-140

Lemus Arturo, Evaluación del rendimiento escolar, Buenos Aires, Ed. Kapelus, 1974 Leyes/reglamentos y lineamientos generales cuarta ed. 1986 Mty. N.L. Méx.

Llarena, R., Mc. Ginn, N et al, " Definición del campo temático de planeación educativa", en Documento Base, Congreso Nacional de Investigación Educativa, vol. I, México 1981.

Manjarez J. Incorporación de prácticas de laboratorio para el desarrollo de la competencia científica explicación de fenómenos Fundación Universidad del Norte Barranquilla Colombia 2017

Marzano, Robert. Dimensiones del aprendizaje: manual para el maestro (2a. ed.). Guadalajara, MX: ITESO - Instituto Tecnológico y de Estudios Superiores de Occidente, 2014. ProQuest ebrary. Web. 11 December 2016. Copyright (C) 2014. ITESO - Instituto Tecnológico y de Estudios Superiores de Occidente. All rights reserved.

Mendoza J., El proyecto ideológico modernizador de las políticas universitarias en México, (1965-1980) Revista Perfiles Educativos, CISE-UNAM, 1981

Padilla A. El Sistema modular de enseñana una alternativa curricular de la educación superior universitaria en México Rev. de docencia Universitaria de educación superior universitaria Vol 10 Oct-Dic 2012, 71-98

Pansza Margarita, Pedagogía y currículo Ed. GERNIKA 1987

Peña S. Apuntes de clase seminario de tesis I, II, III, IV, y V sep 2018, abril 2020 
Phenik, H " Curriculum", en A. Short y D. Marconnit (dirs) Contemporary thought on public school curriculum, Brown Corporation Publishers, Iwoa, 1968.

Rocha j.: La Importancia de la secuenciación de las materias del área de Química del

Dpto. de Química Analítica de la Fac. de Medicina de la U.A.N.L. Julio 1996 (Filosofía y Letras U.A.N.L.)

Rocha j. Análisis Retrospectivo de las Calificaciones obtenidas en el 5to sem de la carrera de Químico Clínico Biólogo Sept. 86 Marzo 92 Oct.1992

Rocha j "Evaluación del error durante la preparación de soluciones: una herramienta para el apoyo de aprendizaje “49 Congreso Mexicano de Química 33 congreso Nacional de educación Química Mérida 2014

Rocha j Guía del alumno Análisis Cuantitativo Escuela y Preparatoria Técnica Médica Universidad Autónoma de Nuevo León Enero 2016

Ruiz M, La formación en competencias Tres procesos metodológicos esenciales, Facultad de Filosofía y Letras Universidad Autónoma de Nuevo León Ed Grafo Print 2008

Taba, H Desarrollo del Curriculum, teoría y práctica, Troquel, Buenos aires, 1976

Taborga, H, "Etapas del proceso de planeación prospectiva universitaria" en Cuadernos de planeación educativa, Dirección General de Planeación, U.N.A.M., México, 1980

Velasco Ugalde. "Notas acerca del diseño curricular, la definición de fases y el Diseño Modular: un ejemplo", en Cuadernos de Formación Docente, D. C. B. S., UAMXoxhumilco, México, 1982

.Zavala A. Once Ideas clave. Como aprender y enseñar competencias. Ed Grao (2008) 17,105

Zavala, JK Historia de la Educación Superior en N.L. 1990 Mty. N.L.

Referencias en línea:

http://www.uanl.mx/sites/default/files/documentos/universidad/historia-de-la-uanl.pdf https://tecnicamedica.uanl.mx/secciones/nuestra_historia.php 